Fig. 4. Wings of Rhydinofoenus kaveahensis, ㅇ, X 36 .

5. Right mandible of Hyptiogaster humeralis Schlett., $q$, as seen from above.

" 6. Right mandible of Pseudofoenus pedunculatus Schlett., o7, as seen from above.

7. Right mandible of Foenus incertus Cresson, as seen from above.

8. Right mandible of Rhydinofoenus kaweahensis n. sp., 우, as seen from above.

Figs. 5 to 8 are of the same degree of magnification.

\title{
Zar Kenntnis einiger paläarktischer Bienen und Beschreibung zweier neuer Arten. (Hym.)
}

Von J. D. Altken, Bremen.

Andrena nigrospina Thoms.

Diese Art ist ohne Zweifel eine ausgeprägte Färbung der A. carbonaria L. (pilipes F.), bei der der Thorax und die Hinterleibsbasis weifsgrau gefärbt sind. Solche Farbenvarietäten sind bei Bremen nicht selten und treten in beiden Geschlechtern, besonders in der zweiten Generation, seltener in der Frühjahrsform auf. Die ovale Grube seitlich an der Basis des zweiten Hinterleibsringes finde ich bei dieser Varietät ebenfalls, wenn auch viel weniger deutlich als bei der Stammform entwickelt. Bei manchen Exemplaren ist sie nur bei starker Vergröfserung zu erkennen, so dafs man wohl verstehen kann, dafs Thom son sie als fehlend bezeichnet. S $\mathrm{chmi}$ edeknecht hat die $A$. nigrospina in seinen "Apidae Europaeae" mit A. Flessae Pz. zusammen hinter A. cineraria L. gestellt. Wegen des grob gerunzelten Mittelfeldes des Mittelsegments hat sie aber neben A. carbonaria L. zu stehen. Alle Arten mit einer so scharf gefurchten Area des Mittelsegments sind meiner Meinung nach zu einer Gruppe, die A. carbonariaGruppe heifsen kann, zu vereinigen. Dahin gehören auch $A$. albicans Müll., Flessae Pz., tibialis K., bimaculata K. und asperrima Pér. Zu A. bimaculata $\mathrm{K}$. ist $A$. Morawitzi Thoms. als rotbeinige Rasse und A. decorata Smith (= Magrettiana Schmied.) als Varietät mit teilweise rot gefärbtem Hinterleib zu rechnen. A. nigrospina Thoms., hat übrigens einen schwach blauschimmernden Hinterleib, Thom son nennt ihn "vix coeruleo micante"; Schmiedeknecht durfte die Art in seiner Tabelle daher nicht unter den Arten mit schwarzem Hinterleib aufführen. (Kennziffern 33 und 47, Seite 442.) 


\section{Nomada errans Lep.}

In einer Sendung Bienen, welche ich von Herrn C. G e i $\int \mathrm{s} l$ e r in Diessen am Ammersee zur Bestimmung erhielt, befand sich auch diese Nomada-Art. Sie wurde bei Diessen auf Angelica silvestris und über Waldboden fliegend gefangen. Für die $q$ war als Fangzeit der 24.-31. VII., für die $\sigma^{7}$ der 24. VII. notiert. Hierdurch ist die Biene meines Wissens zum erstenmal als in Deutschland vorkommend festgestellt worden. Bislang war sie nur von Frankreich bekannt. Eine sehr gute Beschreibung der Art gibt J. Pérez in den "Actes de la Société Linnéenne de Bordeaux", Bd. XXXVII, 1883 , p. 335, wo er auch ausführt, dafs die $N$. vaga Pz. nicht mit $N$. errans Lep., wie Le peletier vermutet, sondern mit $N$. Solidaginis $\mathrm{Pz}$. (= rufipes F.) identisch ist. Ich schliefse mich dieser Ansicht an. Die Zitate bei $N$. vaga $\mathrm{Pz}$. im Kataloge von $\mathrm{Dalla}-\mathrm{T}$ or $\mathrm{re}$ sind also zu $N$. Solidaginis $\mathrm{Pz}$. zu setzen. $N$. errans ist die nächste Verwandte der $N$. rufipes und dürfte oft mit dieser verwechselt werden.

\section{N. rufipes F. (Solidaginis Pz.) 우}

7--9 $\mathrm{mm}$ lang.

Grund der Oberkiefer und Wangen fast immer gelb.

Fühlergeifsel oben vom dritten Gliede an schwarz.

Wangen unten neben dem Clypeus mit einem kleinen dreieckigen gelben Flecken versehen, der sich selten am Augenrande emporzieht.

Flecken des Thorax und Abdomen zitronengelb. Flügelschüppchen zitronengelb.

Mesopleuren und Mesosternum, letzteres hinten mehr oder weniger gelb gefleckt.

Mittelsegment seitlich unterhalb des Mittelfeldes ziemlich dicht und grob punktiert, schwach glänzend.
N. errans Lep.

\section{오}

6,25-7 mm lang.

Grund der Oberkiefer und Wangen rot.

Fühlergeifsel rot, oben nur wenig verdunkelt.

Wangen unten neben dem Clypeus meist ausgedehnt rot gefleckt. Die rote Färbung erstreckt sich am inneren Augenrande als feine Linie meist bis nahe an den oberen Augenrand.

Flecken des Thorax und Abdomen, wenigstens bei den deutschen Exemplaren, weifsgelb. Flügelschüppchen gelbrot.

Mesopleuren und Mesosternum schwarz.

Mittelsegment seitlich unterhalb des Mittelfeldes oben zerstreut und feiner punktiert, unten fast punktlos, stark glänzend. 
Dorsalsegment 4 und Ventralsegment 2-4 mit gelber Binde oder ersteres wenigstens mit gelben Seitenflecken.

Hinterleib oben schwach glänzend, die Segmente dicht und fein, fast bis an das Ende des niedergedrückten Hinterrandes punktiert, von diesem nur der äufserste Rand glatt und punktlos.

Ventralsegmente vorn dicht punktiert.

Hinterschienen am äufseren Spitzenrande mit 2 oder 3 deutlichen, stärkeren und längeren Dörnchen.

\section{$\sigma^{7}$}

$6,25-9,25 \mathrm{~mm}$ lang.

Fühlerschaft unten rot oder gelbrot, Geifsel unten gröfstenteils schwarz, nur die ersten Glieder rötlich.

Oberkiefer mit Ausnahme der Spitze, Oberlippe, Clypeus nicht ganz bis zum Stirnschildchen und die Wangen meist nur unten zitronengelb.

Zeichnung des Thorax und Abdomen zitronengelb.

Mesopleuren gelb gefleckt.

Viertes Dorsalsegment mit einer in der Mitte unterbrochenen, fünftes mit durchgehender gelben Binde.

Ventralsegmente gelb gezeichnet.

Punktierung der Hinterleibsringe oben sehr dicht und fast bis an die Hinterränder reichend.

Hinterschienen ohne schwarze Flecken.
Dorsalsegment 4 und Bauch schwarz, an letzterem die Endränder der Ringe bräunlich durchscheinend.

Hinterleib oben sehr stark glänzend, die Segmente zerstreut und sehr fein, nur bis an den Anfang des niedergedrückten Endrandes punktiert, dieser fast in seiner ganzen Ausdehnung glatt und punktlos, stark glänzend.

Ventralsegmente vorn zerstreut punktiert.

Hinterschienen am äufseren Spitzenrande mit 5 oder 6 deutlichen, schwächeren und kürzeren Dörnchen.

\section{$\sigma^{7}$ \\ $6-6,75 \mathrm{~mm}$ lang.}

Fühlerschaft unten weifsgelb, sämtliche Geifselglieder unten gelbrot, die letzten auch oben rötlich.

Oberkiefer mit Ausnahme der Spitze, Oberlippe, Clypeus und die Wangen fast bis zur Höhe des oberen Augenrandes weifsgelb.

Zeichnung des Thorax und Abdomen weifsgelb.

Mesopleuren schwarz.

Viertes und fünftes Dorsalsegment nur mit kleinen weifsgelben Seitenflecken. Bauch schwarz.

Punktierung der Hinterleibsringe oben weniger dicht, die glatten Hinterränder davon frei.

Hinterschienen aufsen und innen schwarz gefleckt. 
Die beiden Arten lassen sich unschwer unterscheiden. Beide Geschlechter sind an der Hinterleibsfärbung und Punktierung ohne weiteres zu erkennen. N. rufipes hat einen gelb bandierten Bauch und viel glattere Dorsalsegmente, $N$. errans hat einen schwarzen Bauch und viel mattere Dorsalsegmente. Das $q$ von $N$. rufipes ist oben am vierten Hinterleibsring gelb bandiert, das von $N$. errans nicht; das $\sigma^{7}$ von $N$. rufipes hat den vierten und fünften Hinterleibsring gelb bandiert, das von $N$. errans dort nur kleine weilsgelbe Seitenflecke.

Die Wirtsbiene von $N$. errans ist noch nicht bekannt geworden.

\section{Nomada Merceti n. sp.}

ㅇ $9-10 \mathrm{~mm}$ lang. Kopf schwarz, Oberkiefer mit Ausnahme der dunklen Spitze, der vordere Teil des Clypeus, Wangenanhang, der untere Teil der Wangen und ein kleiner runder Fleck am oberen Augenrande, manchmal auch die Oberlippe und ein Streifen der Schläfen am hinteren Augenrand rot. Fühler schwarz, das letzte Geifselglied unten ganz, oben am Ende, der Schaft und die ersten Geifselglieder unten mehr oder weniger rot gefärbt. Zweites Geifselglied etwas länger als das dritte. Oberlippe nach vorn hin mit einem schwachen Zähnchen. Kopf rund, mit dünner greiser Behaarung, Clypeus kaum vorgezogen, vorn abgestutzt, fein und zerstreut punktiert, Gesicht stärker und dichter, Scheitel und Schläfen zerstreuter punktiert. - Am Thorax sind das Pronotum, die Schulterbeulen, ein kleiner Fleck der Mesopleuren, fast das ganze Mesosternum, das Schildchen und das Hinterschildchen und manchmal ein Fleckchen seitlich vor dem Schildchen rot gefärbt. Mesonotum fast kahl, nur hier und da mit einigen grauen Härchen besetzt, schwach glänzend, ziemlich gleichmäfsig und mäfsig stark punktiert. Schildchen schwach gewölbt, in der Mitte schwach gefurcht, glänzend, zerstreut punktiert. Mesosternum stark und mäfsig dicht punktiert, in der Mitte fein gekielt. Mittelfeld des Mittelsegments fein wellig gefurcht, am Grunde etwas stärker, die Seiten des Mittelsegments hinten seitlich mit dichtem, weilsem Haarbüschel. - Hinterleib rot, erster Ring am Grunde schwarz, meist auch der vierte, fünfte und sechste oder nur der fünfte und sechste seitlich am Grunde, manchmal auch die Endränder des dritten und vierten schwarz gefleckt. Die niedergedrückten Endränder der 4 ersten Ringe breit, glatt und punktlos, erster Ring glänzend, nur hier und da mit einem feinen Pünktchen versehen, die übrigen Ringe vor dem niedergedrückten Hinterrande matt, fein und dicht punktiert. Ring 2-6 seitlich mit nach hinten hin stärkeren weifsen Haarhäufchen bekleidet. Bauch rot, die Ringe vom zweiten an am Grunde ziemlich dicht punktiert, die 
niedergedrückten Endränder breit, glatt und punktlos. Beine rot, Mittel- und Hinterhüften, Hinterschenkel und manchmal auch die Hinterschienen mehr oder weniger schwarz gefleckt, Spitze des Aufsenrandes der Hinterschienen mit vielen zi em lich langen schwarzen Dörnchen dicht besetzt. Vorderflügel gebräunt, am Ende der Cubital- und Discoidalzellen hell gefleckt, Flügelschüppchen rot.

○ 9-9,75 mm lang. Dem o ziemlich ähnlich. Kopf schwarz. Die Basalhälfte der Oberkiefer rötlichgelb, die Kiefer dann rot und am Ende schwarz gefärbt, dieser ziemlich stumpf. Oberlippe schwarz, vorn mit schwachem Zähnchen; Wangenanhänge gelb. Gesicht unter den Fühlern dicht und lang silberweifs, Scheitel kurz grau behaart. Fühler schwarz, Geifsel unterseits rotbraun, das letzte Glied fast ganz rot gefärbt, zweites Geifselglied ein wenig länger als das vierte. - Thorax schwarz, Pronotum und Schulterbeulen etwas rotbraun. oben kurz grau, seitlich und besonders unten lang weifs behaart. Mesonotum gleichmälsig dicht und ziemlich stark punktiert. Schildchen ziemlich flach, wenig gefurcht, glänzend, grob und einzeln punktiert. Mittelsegment seitlich locker abstehend behaart, sein Mittelfeld sehr schwach runzlig. - Hinterleib rot, erster Ring an der Basis, dritter bis siebenter seitlich und hinten mehr oder weniger schwarz oder braun gefärbt, zweiter bis vierter seitlich mit rundlichen verschwommenen gelben Flecken, dritter bis fünfter hinten seitlich mit abstehenden weifsen Haarhäufchen. Endplatte schmal, an der Spitze ziemlich tief eingeschnitten. Erster Ring glänzend und zerstreut punktiert, die übrigen Ringe matt und bis auf den glatten Hinterrand dicht und fein punktiert. Bauch rot oder mehr oder weniger braun oder schwarz gefärbt, die Basis der Ringe zerstreut punktiert, die Endhälfte glatt und nicht punktiert. Beine rot, mehr oder weniger, besonders die Hüften, Schenkelringe und die Hinterschenkel schwarz gefärbt, die letzteren am Grunde eingedrückt und $\mathrm{mit}$ langer weifser $\mathrm{Ha}$ a r locke, auch die hinteren Schenkelringe mit etwas kürzerer weifser Haarlocke. Spitze des Aufsenrandes der Mittel- und Hinterschienen gelblichrot gelbrot gefärbt, die Bedornung der letzteren ist ähnlich, aber nicht so stark, wie beim ․ Flügel etwas schwächer gebräunt, als beim ㅇ.

Die vorliegende Art, welche Herrn Dr. R. G. Mercet in Madrid, der sich um die Erforschung der Sphegiden- und Chrysidenfauna Spaniens grofse Verdiente erworben hat, zu Ehren benannt wurde, ist die nächste Verwandte der nord- und zentraleuropäischen $N$. similis Mor. Mit dieser Art hat das $q$ vor allem das fast kahle Mesonotum, das $\sigma^{r}$ die gekrümmte Haarlocke an 
der Basis der Hinterschenkel gemein. Beide Arten ähneln sich auch im äufseren Habitus, das of der $N$. Merceti unterscheidet sich aber von der $N$. similis sofort durch die auffallende schwarze Bedornung an der Spitze des Aufsenrandes der Hinterschienen und rot gefärbtes Schildchen und Hinterschildchen, das $\sigma^{7}$ durch die stärkere Bedornung der Hinterschienen. Auch die Gröfse bietet bei beiden Geschlechtern ein gutes Trennungsmerkmal.

In der $N$. Merceti vermute ich den Schmarotzer einer der vielen in Spanien vorkommenden Pamurgus-Arten, worauf auch die Fangzeit für das eine $q$ hindeutet.

Es lagen zur Untersuchung eine ziemliche Zahl von Exemplaren vor, welche bei Madrid, Alicante (Mercet), Pozuelo de Calatrava, La Fuente und Villalba (D us met), an letzterem Orte 1 o am 17. VII. 1901, gefangen wurden.

\section{Halictus Duckei n. sp.}

ㅇ $4 \mathrm{~mm}$ lang. Erzgrün. Kopf langgestreckt, ähnlich wie bei H. Smeathmanellus K. gebildet, aber schmaler; dünn, am Vorderrand des Clypeus abstehend und ungleich lang, am Hinterrand des Scheitels kurz und gleichlang greis behaart. Clypeus vorgezogen, zerstreut, vorn ziemlich stark punktiert, hier blauschwarz gefärbt und etwas kupferfarben glänzend. Stirnschildchen gewölbt, zerstreut, der übrige Kopf fein und gleichmälsig dicht punktiert. Fühlergeifsel unterseits rotbraun. - Mesonotum aufserordentlich fein lederartig gerunzelt und aufserdem dicht und fein punktiert. Schildchen flach, ziemlich dicht punktiert. Hinterschildchen mehr oder weniger dicht abstehend behaart. Mittelfeld des Mittelsegments fein und dicht wellig längs-, dazwischen ein wenig quergerunzelt, hinten fein und scharf, aber unterbrochen gerandet. - Hinterleibsringe mit gelbrot durchscheinenden Endrändern. Erster Hinterleibsring stark g 1 ä n z e n d, fein und zerstreut punktiert, am niedergedrückten Hinterrande punktlos, wie die übrigen Ringe, die vorn aber dicht punktiert sind. Alle Ringe seitlich mit abstehenden, weifslichen Härchen dünn bekleidet, $\mathrm{z}$ weiter und dritter Ring an der Basis weifs befilzt (bei eingezogenen Ringen nur seitlich sichtbar), vierter Ring ganz mit anliegenden weifsen Härchen dicht bekleidet. Endfurche rötlich durchscheinend; seitlich dicht greis behaart. - Beine schwarz, nur die Kniee und letzten Tarsenglieder etwas rötlich. Bürste schwach entwickelt, silbergrau. Flügel glashell, mit hellgelben Adern und sehr hellem Stigma.

In der Form des Mittelfeldes am Mittelsegment schliefst sich diese kleinste der ganz grünen Halictus-Arten eng an H. Smeathmanellus $\mathrm{K}$. an. Nahe dürfte ihr der mir nur nach der Beschreibung 
bekannte $H$. annulipes Mor. von Turkestan stehen, der aber anders gefärbte Beine und einen fast ovalen Kopf hat. Wegen des gerandeten Mittelfeldes am Mittelsegment (Metathorax bei Morawitz) gehört diese Art auch in die Gruppe des Smeathmanellus.

Ich fing 4 Exemplare dieser zierlichen Art in der Nähe von Triest beim Aufstieg zum Mte. Spaccato. Sie ist Freund A. Ducke in Pará, der zur Erforschung der Apidenfauna von Triest viele wertvolle Beiträge lieferte, zu Ehren benannt worden.

$$
\text { Halictus Perezi Alfk. }
$$

o7 $7 \mathrm{~mm}$ lang. Dem Weibchen, welches in "Ztschr. f. Hym. u. Dipt. ", 1907, p. 203, beschrieben wurde, ähnlich. Schwarz, Fühler kurz, von Thoraxlänge, die Geifsel unterseits vom zweiten Gliede an rotbraun. Gesicht dicht weifsfilzig. Clypeus wenig vorgezogen, vorn weifsgelb gefärbt. Thorax weifsgrau behaart. Mittelfeld des Mittelsegments wie beim $q$ fein und gerade längsgerieft. Area interna hinten abgerundet, gekörnt. Erster Hinterleibsring ziemlich dicht und gleichmäfsig, am Endrande etwas zerstreuter, vierter und fünfter viel feiner und zerstreuter punktiert, zweiter, dritter und vierter am Grunde seitlich weifs gefilzt. Alle Schienen am Grunde und an der Spitze, die vorderen auch innen, gelblich, alle Tarsen gelb, die letzten mehr rötlich gefärbt. Endränder der Bauchringe rötlichbraun, Flügelmal dunkelbraun gefärbt.

Von Halictus sexnotatulus Nyl. läfst sich dieses $\sigma^{\top}$ durch die gelblich gefärbten Schienen und Tarsen (bei H. sexnotatulus schwarz) and den punktierten Hinterleib (bei $H$. sexnotatulus fast punktlos) und von $H$. fallax Mor. durch das dunkelbraune Flügelmal (bei H. fallax hellgelb) unterscheiden.

Mir liegt 1 Exemplar aus Spanien vor.

\section{On some undescribed Indian Bees (Tetralonia, Megachile and Halictus). (Hym.)}

By P. Cameron, New Mills by Stockport (England).

$$
\text { Tetralonia pruinosa sp. nov. }
$$

Black, the flagellum of antennae for the greater part dark rufous, the head, pleurae and base of abdomen densely covered with white, the top of thorax with dark pale fulvous pubescence, the top of abdomen densely pruinose, the sides and apical segments densely covered with glistening white hair, the ventral hair white, 


\section{$2 \mathrm{BHL}$ Biodiversity Heritage Library}

Altken, J D . 1909. "Zur Kenntnis einiger paläarktischer Bienen und Beschreibung zweier neuer Arten. (Hym.)." Deutsche entomologische Zeitschrift 1909(1), 41-47. https://doi.org/10.1002/mmnd.48019090107.

View This Item Online: https://www.biodiversitylibrary.org/item/103377

DOI: https://doi.org/10.1002/mmnd.48019090107

Permalink: https://www.biodiversitylibrary.org/partpdf/235932

\section{Holding Institution}

Harvard University, Museum of Comparative Zoology, Ernst Mayr Library

\section{Sponsored by}

Biodiversity Heritage Library

\section{Copyright \& Reuse}

Copyright Status: Public domain. The BHL considers that this work is no longer under copyright protection.

This document was created from content at the Biodiversity Heritage Library, the world's largest open access digital library for biodiversity literature and archives. Visit BHL at https://www.biodiversitylibrary.org. 